\title{
Sixteen Years of Terra MODIS On-orbit Operation, Calibration, and Performance
}

\author{
X.Xiong ${ }^{1}, A$. Angal $^{2}, A . W u^{2}$, D. Link ${ }^{2}, X$. Geng $^{2}, W$. Barnes $^{3}$, and V. Salomonson $^{4}$ \\ ${ }^{1}$ Sciences and Exploration Directorate, NASA/GSFC, Greenbelt, MD 20771, USA \\ ${ }^{3}$ Science Systems and Applications Inc., 10210 Greenbelt Road, Lanham, MD 20706, USA \\ ${ }^{2}$ University of Maryland, Baltimore County, 1000 Hilltop Circle, Baltimore, MD 21250, USA \\ ${ }^{4}$ University of Utah, Salt Lake City, UT 84112, USA
}

\begin{abstract}
Terra MODIS has successfully operated for more than 16 years since its launch in December 1999. From its observations, many science data products have been generated in support of a broad range of research activities and remote sensing applications. Terra MODIS has operated in a number of configurations and experienced a few anomalies, including spacecraft and instrument related events. MODIS collects data in 36 spectral bands that are calibrated regularly by a set of on-board calibrators for their radiometric, spectral, and spatial performance. Periodic lunar observations and long-term radiometric trending over well-characterized ground targets are also used to support sensor on-orbit calibration. Dedicated efforts made by the MODIS Characterization Support Team (MCST) and continuing support from the MODIS Science Team have contributed to the mission success, enabling wellcalibrated data products to be continuously generated and routinely delivered to users worldwide. This paper presents an overview of Terra MODIS mission operations, calibration activities, and instrument performance of the past 16 years. It illustrates and describes the results of key sensor performance parameters derived from on-orbit calibration and characterization, such as signal-to-noise ratio (SNR), noise equivalent temperature difference (NEdT), solar diffuser (SD) degradation, changes in sensor responses, center wavelengths, and band-to-band registration (BBR). Also discussed in this paper are the calibration approaches and strategies developed and implemented in support of MODIS Level 1B data production and re-processing, major challenging issues, and lessons learned.
\end{abstract}

Key words: Terra, MODIS, Calibration, Solar Diffuser, Blackbody, Stability Monitor, Moon, SRCA

\section{INTRODUCTION}

The Terra satellite, formerly called EOS AM-1, is the flagship of NASA's Earth Observing System (EOS), launched on December 18, 1999. It has successfully operated for more than 16 years. Terra carries five sensors designed to observe the Earth's atmosphere, cryosphere, land, oceans, and energy budget. To date, an unprecedented amount of data has been collected to support various scientific studies and applications. MODIS is a key instrument on the Terra spacecraft. It was developed and built with improvements over a number of heritage sensors, in terms of its spectral range, spatial resolution, and temporal coverage. MODIS collects data in 36 spectral bands with wavelengths ranging from 041 to $14.4 \mu \mathrm{m}$ and at 3 spatial resolutions (nadir): $250 \mathrm{~m}$ for bands $1-2,500 \mathrm{~m}$ for bands $5-7$, and $1 \mathrm{~km}$ for bands 8-36. It is a scanning radiometer incorporating a two-sided scan mirror with an Earthviewing scan angle range of $\pm 55^{\circ}$ relative to the instrument nadir. Thanks to its large swath and sun-synchronous orbit, MODIS can achieve complete global coverage in less than 2 days ${ }^{1}$. Through its entire mission, nearly 40 science data products have been routinely generated from MODIS observations and distributed to users worldwide, supporting a broad range of research and operational activities ${ }^{2}$. 
MODIS spectral bands 1-19 and 26 are the reflective solar bands (RSB), covering wavelengths from 0.41 to $2.2 \mu \mathrm{m}$. The remaining bands, 20-25 and 27-36, are referred to as the thermal emissive bands (TEB), covering wavelengths from 3.7 to $14.4 \mu \mathrm{m}$. MODIS spectral bands (and detectors) are located on four focal plane assemblies (FPA): visible (VIS), near infrared (NIR), short- and mid-wave infrared (SMIR), and long-wave infrared (LWIR). The VIS and NIR FPA are operated without temperature control, whereas the SMIR and LWIR FPA temperatures are controlled, nominally at $83 \mathrm{~K}$, via a passive radiative cooler. Because of this, the SMIR and LWIR FPA are also referred to as the cold FPA (CFPA). Compared to its heritage sensors, MODIS was developed with stringent calibration requirements over its entire mission. In addition to comprehensive pre-launch calibration efforts made to examine, characterize, and verify sensor performance, the MODIS instrument carries a set of on-board calibrators $(\mathrm{OBC})$ that are capable of performing various calibration functions during its on-orbit operation. The $\mathrm{OBC}$ include a solar diffuser (SD), a solar diffuser stability monitor (SDSM), a blackbody (BB), and a spectro-radiometric calibration assembly (SRCA) $)^{3}$.

From launch to present, Terra MODIS has operated in a number of configurations. It is currently operated using the A-side electronics with its B-side formatter. It has also experienced a few spacecraft and instrument related anomalies or events (detailed in the next section). Still, the instrument itself and its OBC continue to operate and function normally. The dedicated efforts made by the MODIS Characterization Support Team (MCST), with support from the Mission/Flight Operation Team (MOT/FOT) and the MODIS Science Team (MST), have greatly contributed to the MODIS mission success.

In the following, we present an overview of Terra MODIS operations, its calibration activities, and instrument overall performance over the past 16 years. Specific results of key sensor performance parameters derived from instrument $\mathrm{OBC}$ are described and illustrated. Remaining challenging issues and future improvements are also discussed in this paper, along with the lessons learned from 16 years of Terra MODIS operation and calibration.

\section{OPERATIONS AND CALIBRATION ACTIVITIES}

Since launch, Terra MODIS has experienced several changes of its operational configurations, which are related to the use of its on-board calibrators, electronics, and data formatters. Table 1 provides a summary of Terra MODIS instrument operations, detailing major events such as spacecraft launch and sensor's first light, and its key operational configuration changes. On-orbit operation configurations of Terra MODIS include all A-side (electronics and data formatter), all B-side, and A-side electronics with B-side formatter. For the last 14 years, from September 17, 2002 to present, the same configuration with A-side electronics and B-side formatter has been used.

Due to the loss of instrument radiative cooler thermal margin, Terra MODIS CFPA were briefly set at $85 \mathrm{~K}$, instead of its initial setting of $83 \mathrm{~K}$. The cooler's thermal margin was later recovered following an outgas operation. Consequently, the CFPA temperature control was set back to $83 \mathrm{~K}$. A major change to SD/SDSM calibration process was made on July 2, 2013 to permanently fix the SD door at the open position. This was the result of an SD door operation anomaly. Before this, the SD calibration was scheduled on a regular basis and the SD door was opened only during scheduled calibration events. Since the SD door was fixed in the open position, SD calibration data are available every orbit and increased solar exposure of the SD panel has led to relatively large SD degradation. Meanwhile, the SDSM operation continues on a regular basis with gradually-reducing frequencies over time. In addition to a thermal source, the SRCA has four 10-W and two 1-W lamps in its integration sphere. Currently, two of its four 10-W lamps have failed to operate normally thus been taken out of service, one in 2004 and another in 2006, respectively. Because of this, several SRCA operation scripts and commands had to be modified in order to maintain its key calibration functions.

While most of the major events occurred during the first six years into the mission, the latest safe mode of the MODIS instrument was on February 18, 2016. This was caused by the spacecraft safe-mode triggered during its planned inclination adjustment maneuver (IAM). When the Terra spacecraft entered safe mode (2016/049: 14:33:17Z), it caused the MODIS and other instruments on-board to enter safe mode as well. Because of this, MODIS nadir and space-view doors were closed while the SD door was left open as planned since July, 2003. Following a series of investigations, analyses, and simulated testing, the Terra Flight Operations Team (FOT) successfully recovered the spacecraft from its safe mode to the normal operation mode on February 22 (2016/053). 
Shortly after the spacecraft went back to its nominal operation, the MODIS Instrument Operations Team (IOT) also brought the MODIS sensor back to its normal science mode, following an outgas operation. The nadir door was opened on February 24, 2016 (2016/055 20:01:32) to resume the earth observations.

In order to examine the functionality of the $\mathrm{OBC}$ and instrument overall performance, several out-of-turn calibration activities were scheduled and implemented following the safe mode recovery. Additionally, the instrument telemetry was closely monitored. All the key telemetry temperatures (instrument and focal plane) returned to expected values and fully stabilized by day 2016/058. Based on careful evaluation from MCST and MODIS science teams, sensor data, starting from 2016/059:0000, is valid for downstream science products.

Table 1. Key Terra MODIS Operational Configuration Changes

\begin{tabular}{cl}
\hline Date & \multicolumn{1}{c}{ Event Description } \\
\hline $12 / 18 / 99$ & Terra launch \\
\hline $02 / 13 / 00$ & Science Mode, A-side electronics \\
\hline $02 / 24 / 00$ & First light; nadir aperture door open \\
\hline $06 / 08 / 00$ & Cold focal plane assembly stopped controlling temperature \\
\hline $08 / 03 / 00$ & Focal plane assembly temperature set to $85 \mathrm{~K}$ \\
\hline $08 / 05 / 00$ & Formatter reset anomaly; MODIS enters standby mode, then safe mode \\
\hline $10 / 30 / 00$ & MODIS switches to B-side electronics configuration \\
\hline $06 / 15 / 01$ & Power supply 2 (PS2) B-side shutdown (Safe Mode) \\
\hline $07 / 02 / 01$ & MODIS switches to A-side electronics configuration using PS1 \\
\hline $03 / 19 / 02$ & Spacecraft safe mode hold anomaly during maneuver \\
\hline $09 / 17 / 02$ & Switch to B-side formatter; other components remain on A-side \\
\hline $05 / 06 / 03$ & Solar diffuser door fails to open when commanded \\
\hline $07 / 02 / 03$ & Solar diffuser door set to remain open with screen down \\
\hline $12 / 16 / 03$ & Attitude Control Electronics anomaly; S/C transition to safe mode \\
\hline $11 / 22 / 04$ & SRCA 10W lamp \#2 fails to operate normally \\
\hline $02 / 18 / 06$ & SRCA 10W lamp \#3 becomes abnormal and is taken out of service \\
\hline $08 / 22 / 06$ & Nadir aperture door and SV door inadvertently closed (re-opened 2.5 hrs. late) \\
\hline $02 / 18 / 16$ & Spacecraft safe hold anomaly during IAM \#43 (caused MODIS to safe mode) \\
\hline $02 / 24 / 16$ & MODIS nadir door open \\
\hline
\end{tabular}

MODIS on-orbit calibration approaches are illustrated in Figure 1. The SD/SDSM system is primarily used for the RSB calibration, which is reflectance based via reference to its on-board SD bidirectional reflectance factor (BRF). The on-board BB is nominally controlled at a constant temperature (290 K for Terra MODIS), serving as the calibration reference for the TEB. Except during early-mission, a BB warm-up and cool-down (WUCD) operation has been performed on a quarterly basis, during which the BB temperature can vary from instrument ambient to 315 $\mathrm{K}^{3-5}$.

The SRCA can be operated at different modes with built-in sources and limited internal calibration capability. It is mainly used to characterize instrument spatial and spectral performance ${ }^{3,6,7}$. In addition to OBC measurements, periodic lunar observations and long-term radiometric trending over well-characterized ground targets are made to supplement sensor on-orbit calibration and characterization ${ }^{8-10}$. MODIS was also designed with a functional self-test process, called electronic calibration (ECAL) that can be applied to track electronic responses or gains ${ }^{11}$. Since launch, Terra MODIS ECAL for the photovoltaic (PV) spectral bands (1-30) has also been performed regularly.

Table 2 provides a summary of all key OBC activities, including regularly scheduled SD/SDSM calibration events, BB temperature WUCD events, and SRCA operations in its radiometric, spatial and spectral modes. Regularly scheduled lunar calibrations and ECAL are also included. In the summary, the SD/SDSM calibrations with SD 
screen open and SD screen down are counted separately. Measurements from these calibration activities provide vital information that can be used to evaluate the instrument's radiometric, spatial, spectral, and electronic performance.

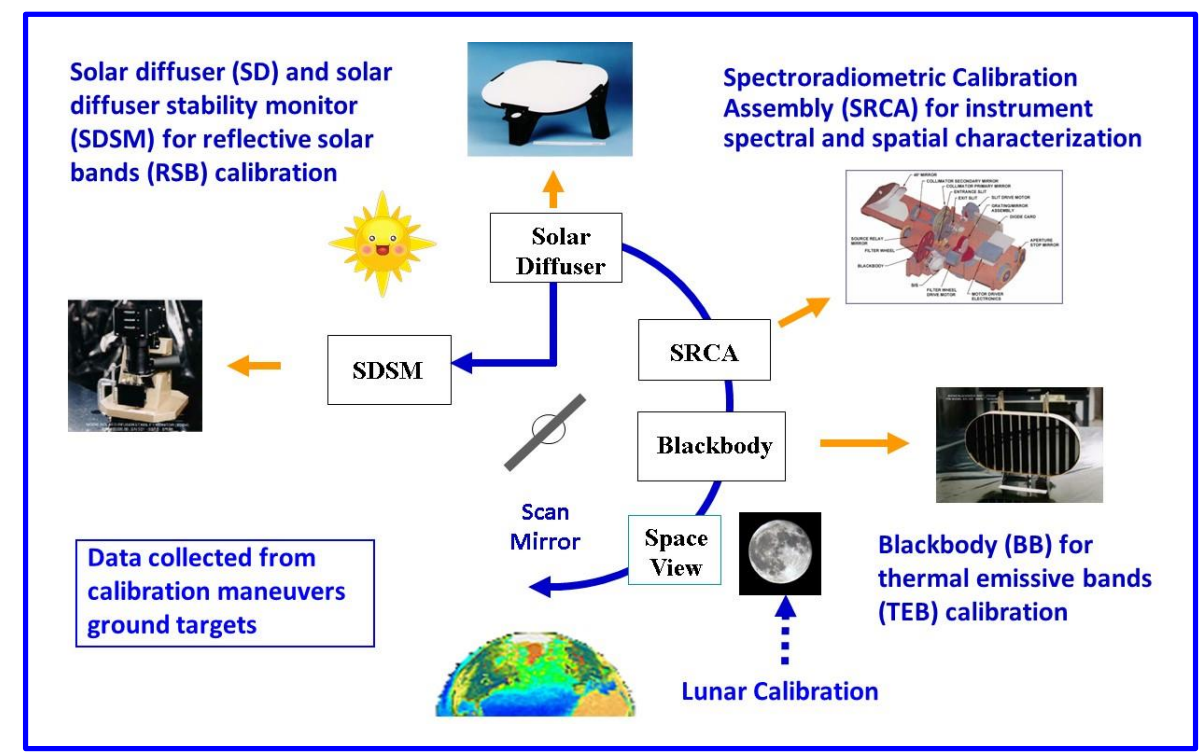

Figure 1. MODIS on-orbit calibration and characterization methodologies.

Table 2. Summary of activities of the on-board calibrators as of September 1, 2016

\begin{tabular}{|c|c|c|c|c|c|c|}
\hline SD/SDSM & BB & Lunar Cal & ECAL & SRCA (rad) & SRCA (spat) & SRCA (spec) \\
\hline 719 & 101 & 165 & 81 & 208 & 89 & 63 \\
\hline
\end{tabular}

\section{ON-ORBIT PERFORMANCE}

\section{Instrument and $O B C$}

As examples of key MODIS telemetry, Figure 2 shows the multi-year trending of instrument and focal plane temperatures. While the operating temperatures of the SMIR and LWIR FPA (CFPA) are constantly controlled, the VIS and NIR FPA (warm FPA) are not. The warm FPA temperatures generally follow the trending of instrument temperature. As presented in Figure 2 (left panel), only a small increase of approximately $3.5 \mathrm{~K}$ in the instrument and VIS FPA temperature is observed over 16 years of Terra MODIS on-orbit operation. With the exception of initial instrument-related events (loss of CFPA temperature control), the CPFA temperatures have been relatively stable over the mission lifetime. After the recent safe hold event, a small change of about $0.03 \mathrm{~K}$ has also been noticed in the SMIR FPA temperatures. Details of the safe-mode impact on the instrument performance will be discussed in the next section of this paper.
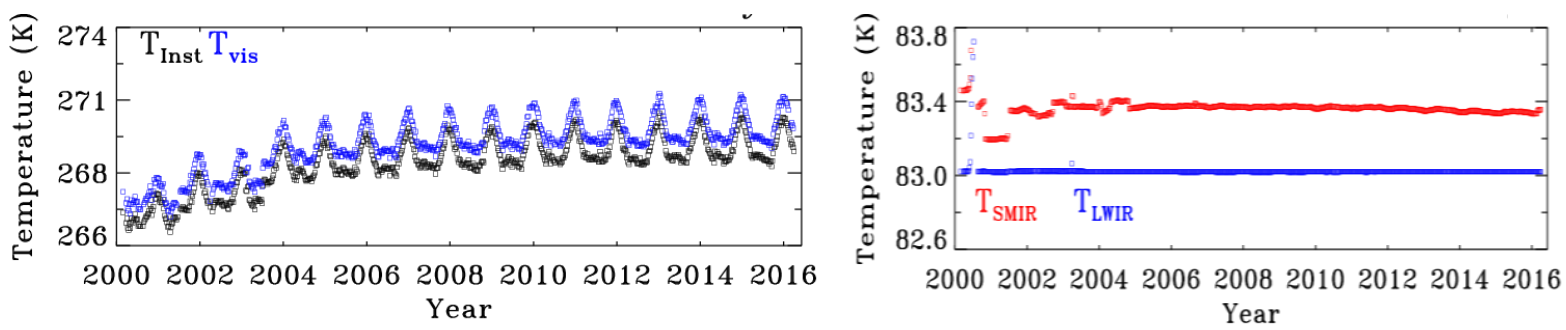

Figure 2. Key Terra MODIS telemetry trending: instrument and VIS FPA temperature (left) and SMIR and LWIR FPA temperature (right). 
The lifetime trending of Terra MODIS on-board BB temperature is presented in Figure 3 (left panel). The data used in the plot are weekly averaged temperatures when the BB is normally controlled at $290 \mathrm{~K}$. The temperatures during instrument special operations, calibration events, and anomalies are not included in the trending plot. For example, a BB WUCD operation is performed periodically over the entire mission, during which the BB temperature varies from instrument ambient temperature to $315 \mathrm{~K}$. For a brief period in early mission, the instrument was configured to B-side electronics and the BB was operated at a slightly higher temperature by about $25 \mathrm{mK}$. Since late 2003, the instrument has been operated using A-side electronics and B formatter configuration. The lifetime trending of the $\mathrm{BB}$ has been relatively stable with a long-term variation of approximately $15 \mathrm{mK}$.

On-orbit changes of instrument on-board SD BRF are routinely monitored via regularly scheduled SDSM calibrations ${ }^{4}$. Examples of Terra MODIS SD degradation trending for select SDSM detectors (equivalently the wavelengths) are also shown in Figure 3 (right panel). Since July $2^{\text {nd }}, 2003$ the SD door has been fixed at an open position due to an anomaly. As a result the SD degradation rates have significantly increased as a result of more frequent solar exposure. To date, the SD BRF has decreased about $50 \%$ at $0.41 \mu \mathrm{m}, 25 \%$ at $0.53 \mu \mathrm{m}, 14 \%$ at 0.64 $\mu \mathrm{m}$, and $5 \%$ at $0.85 \mu \mathrm{m}$. The SD/SDSM system is used primarily for the RSB on-orbit calibration. The calibration coefficients derived from SD observations must be corrected for the changes in the SD BRF.
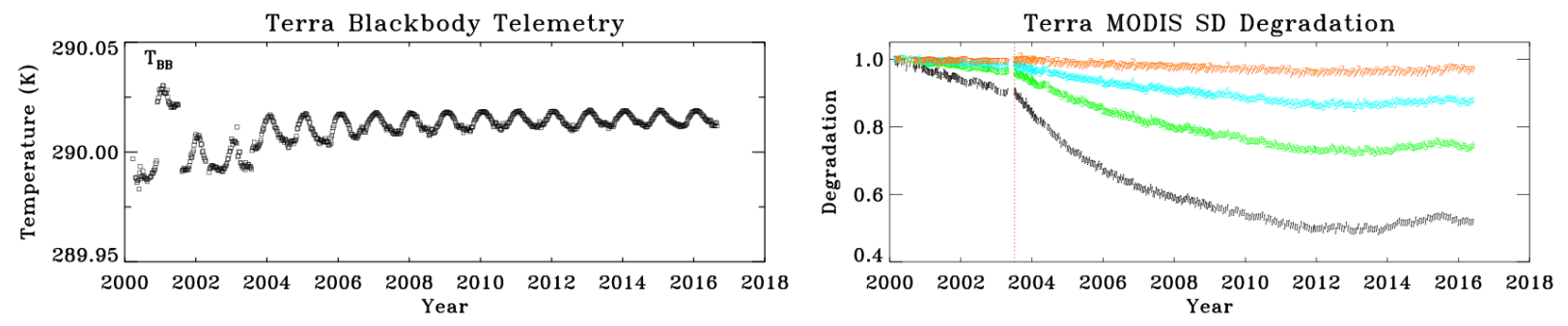

Figure 3. Lifetime trending of Terra MODIS on-board BB temperature (left) and trending of SD on-orbit degradation at selected wavelengths (right).

\section{Radiometric Performance}

The changes in the RSB responses are tracked on-orbit using regular SD/SDSM and lunar observations ${ }^{4,8}$. In general, the VIS spectral bands with shorter wavelengths have experienced larger changes and are also known to exhibit large mirror side and scan angle dependence. Figure 4 (left panel) shows the response (or gain) trending for bands 8 and 12 from regular SD (denoted by lines) and lunar (denoted by symbols) measurements. The measurements are detector-averaged and normalized to their first on-orbit measurements. A similar gain trending is displayed in Figure 4 (right panel) for the NIR spectral bands 1 and 2, showing a gain increase from launch to 2010. While the change for most NIR bands is within $10 \%$, the largest gain change for the VIS bands has reached up to $50 \%$. Overall trending profiles from SD and lunar observations are similar but the amount of changes are different, especially for VIS bands, as SD and lunar observations are made at different angles of incidence (AOI) to the scan mirror. After the most recent safe hold event, the gain change for the VIS and NIR bands has been less than $1 \%$. The SWIR bands located on the CFPA show a gain change of up to $2 \%$.
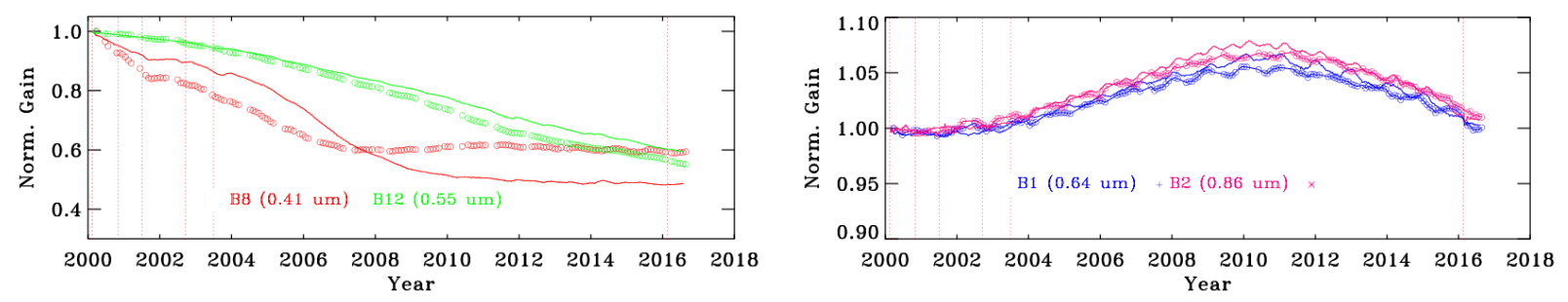

Figure 4. Examples of normalized gain (response) trending for Terra MODIS VIS bands 8 and 12 (left) and NIR bands 1 and 2 (right) from SD observations (shown in lines) and lunar observations (shown in symbols). Vertical lines indicate changes of instrument operation configurations and major events.

MODIS TEB are calibrated by the on-board BB ${ }^{5}$. Overall responses of Terra MODIS MWIR bands (20-25) are very stable. Except for the differences caused by different operation configurations and CFPA temperatures, the long- 
term gain changes are generally within $2-3 \%$ on a band-averaged basis. This is illustrated in Figure 5 (left panel) for bands 20 and 25. Since launch, the LWIR PV bands 27-30 have experienced 10 to $20 \%$ drop in detector gains with the largest changes in bands 27 and 29. Most of the changes are related to on-changes of electronic crosstalk in these bands. On the other hand, the LWIR photoconductive (PC) bands 31-36 are very stable to within 1.5\%. Examples of LWIR gain changes are presented in Figure 5 (right panel) for bands 27 and 36.

Due to the most recent Terra safe hold event, some of the LWIR PV detectors have large decrease in detector gains (up to 18\%). By comparison, the impact on other TEB is generally small. This is illustrated in Figure 6 using TEB linear calibration coefficients, which are inversely proportionally to detector gains, for bands 25 and 27 . The calibration coefficients were derived from on-board BB measurements on a scan-by-scan basis for mirror side 1 over two orbits, one before and one after the latest Terra spacecraft safe hold event.
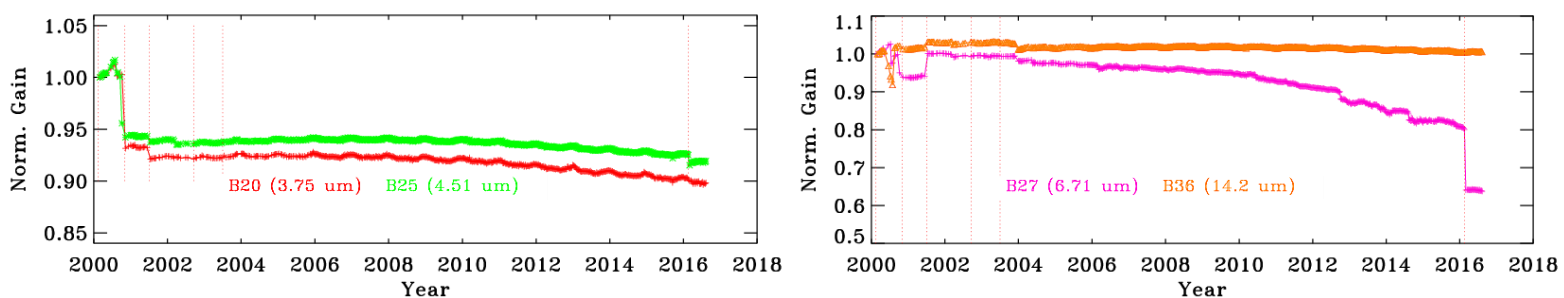

Figure 5. Examples of normalized gain (response) trending for Terra MODIS MWIR bands 20 and 25 (left) and LWIR bands 27 and 36 (right). Vertical lines indicate changes of instrument operation configurations and major events.
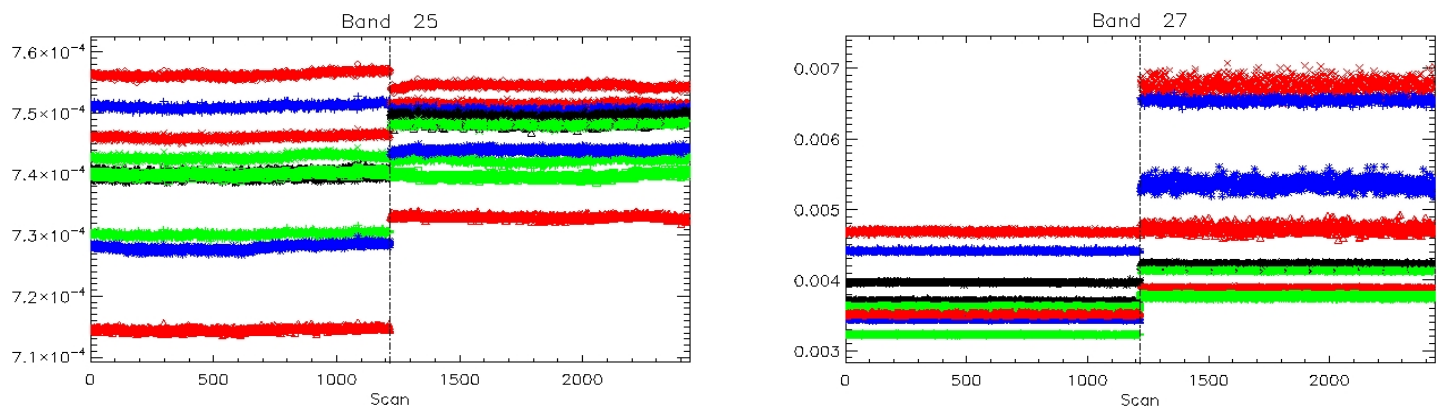

Figure 6. Short-term stability (scan-by-scan detector calibration coefficients) of Terra MODIS bands 25 and 27 . Two orbits of data from mirror side 1 observations were used in each plot with one before and one after the safe hold event (separated by the vertical line in the middle).

MODIS 36 spectral bands have a total of 490 detectors. For the RSB, the detector noise specification is described in terms of its signal to noise ratio (SNR). For the TEB, it is expressed in terms of its noise equivalent temperature difference (NEdT). The sensor design requirements are specified at the typical radiances or temperatures. For Terra MODIS, 30 detectors (20 in band 7 and 10 in band 36) failed to meet specified noise characterization requirements, thus being identified as noisy detectors. On-orbit SNR and NEdT are constantly monitored using OBC observations ${ }^{12,13}$. At launch, 35 detectors were considered as the noisy detectors. Now after more than 16 years of on-orbit operation, Terra MODIS has 53 noisy detectors. In addition, 3 detectors (one in band 29 and two in band 30) are currently flagged as inoperable. It should be pointed out that most noisy and inoperable detectors occurred after launch are located in the LWIR PV bands and nearly half of them were caused by the recent safe hold event.

\section{Spatial and Spectral Performance}

Although SRCA can be operated in 3 different modes (radiometric, spatial, and spectral), its primary design objective is to provide instrument on-orbit spatial and spectral calibration capability ${ }^{6,7}$. Key spatial performance parameters derived from SRCA measurements include band-to-band registration (BBR) and modulation transfer function (MTF). Key spectral performance parameters tracked on-orbit are changes in spectral band center wavelengths $(\mathrm{CW})$ and bandwidths $(\mathrm{BW})$. Since launch, Terra MODIS spatial and spectral performance has been characterized regularly by operating SRCA in its spatial and spectral modes, respectively. The spatial calibration can be made for all spectral bands (TEB and RSB), whereas the spectral calibration can be made only for the VIS and 
NIR spectral bands due to limitations of its internal sources and spectral reference. In general, Terra MODIS spatial performance has been stable over time.

Figure 7 shows its on-orbit BBR in the along-scan direction. Results shown are yearly averaged for 2001, 2005, 2010, and 2015. Except for a couple of band pairs (e.g. band 30 and 32), the BBR performance continue to meet the design requirements $( \pm 100 \mathrm{~m})$ in both along-scan and along-track direction. Displayed in Figure 8 are the on-orbit changes in spectral band center wavelengths (band averaged). For most VIS/NIR bands, CW changes are within 0.5 $\mathrm{nm}$. Similarly, on-orbit changes in BW are generally within $1.0 \mathrm{~nm}$. A few relatively large changes are observed for bands with relatively broad bandwidths.

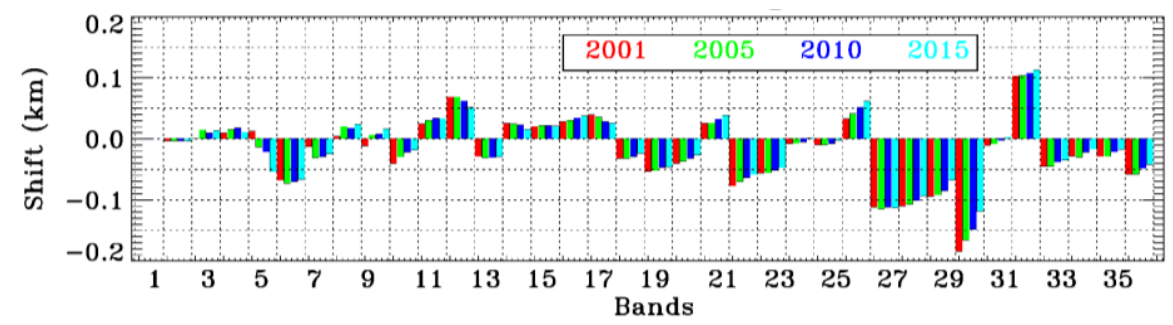

Figure 7. Terra MODIS on-orbit band-to-band registration (BBR) in along scan direction (yearly averaged for 2001, 2005, 2010, and 2015).

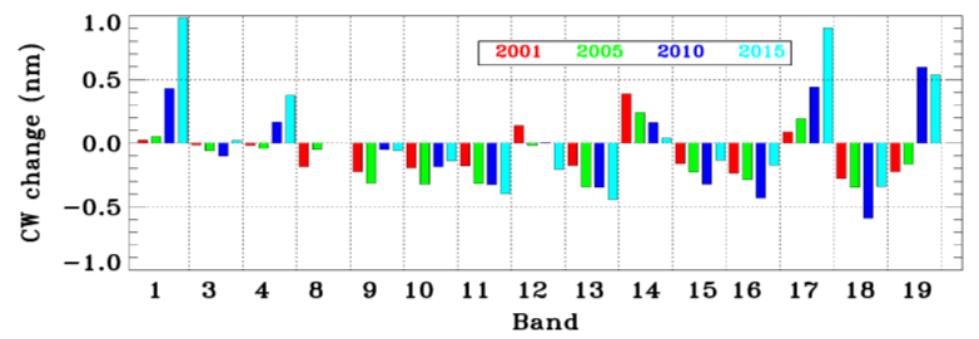

Figure 8. Terra MODIS on-orbit changes in spectral band center wavelengths (yearly averaged for 2001, 2005, 2010, and 2015).

\section{CHALLENGING ISSUES AND FUTURE IMPROVEMENTS}

As Terra MODIS continues to operate beyond its designed life of 6 years, a sustained effort is required to carefully monitor the instrument performance and to address various challenging issues. In addition to instrument operation support, the MCST is also responsible to maintain and improve the L1B software as well as to develop calibration approaches. The current operational versions of the MODIS L1B include both Collection 5 (C5) and Collection 6 (C6). The $\mathrm{C} 5$ has been overlapping with $\mathrm{C} 6$ for more than 3 years and is expected to end its production at the end of 2016.

Although the C6 incorporates improved algorithms (for both RSB and TEB) as compared to C5, several new enhancements have been identified thereafter ${ }^{14}$. While some enhancements have already been incorporated in the forward C6 L1B, others are still under science evaluation. The following are some of the changes in the calibration algorithms that have been incorporated since C6.

- Terra band 10 EV-based RVS: In C6, the measurements for the on-board calibrators were supplemented with measurements from pseudo-invariant desert sites to characterize the scan-angle dependence. At the beginning of C6, this approach was implemented to Terra bands $1-4,8$ and 9 over the entire mission. The long-term observations of the desert sites revealed an evolving reflectance drift in Terra band 10, which at the time relied on the RVS solely derived from the on-board calibrators. Furthermore, the high-gain band 10 saturated while observing these "bright" desert sites. A modified approach that relied on the lower-radiance Algerian desert site to supplement the on-board calibrators was formulated and implemented. This change was applied in the C6 L1B, starting from February 2014. An entire mission reprocess that includes this change was provided to NASA's Ocean Biology Processing Group (OBPG) to help generate consistent long-term ocean color products. 
- Terra SWIR band SD degradation correction: The SDSM tracks the SD degradation from 0.41 to $0.936 \mu \mathrm{m}$ and no correction for SD degradation is applied to the MODIS SWIR bands $(1.2 \mu \mathrm{m}$ and beyond). After C6 went into production, the long-term reflectance trending from the pseudo-invariant desert sites indicated an upward drift of $1.2 \%$ in Terra band 5. Following an independent verification, an EV-based correction was developed and applied to Terra MODIS band 5 to minimize this long-term drift. A similar drift with relatively small magnitude was also seen to evolve in the trending using the deep convective clouds (DCC) for Terra band 26. As a result, a correction for band 26 has also been applied in the $\mathrm{C} 6$ forward processing.

- Terra PV LWIR crosstalk: The crosstalk correction has been significantly improved based on recent science tests using globally distributed granules in 2015. The new improvements in the algorithm include adding the crosstalk contribution from in-band neighboring detectors and separating particular detectors of the sending bands. It has demonstrated that the current algorithm works consistently before and after the recent Terra safe mode. Currently, the Terra PV LWIR crosstalk correction algorithm has been applied to a testing version of L1B code and delivered for more vigorous science testing via a large-scale data set.

- Electronic crosstalk: Due to the impact of electronic crosstalk, the update of a0/a2 is conducted quarterly following each BB WUCD operation for the LWIR PV bands (27-30). This is currently applied to the testing version of L1B.

Although it has been more than a decade beyond its design lifetime, the MODIS instrument on the Terra platform has demonstrated good performance over its entire mission. As instrument ages, an intensive review of the strategies and methodologies employed in the calibration process is necessary. Tracking on-orbit changes in the sensor RVS, especially at shorter wavelengths, has been a major challenge for the RSB calibration. The C6 on-orbit RVS approach is an improvement over its previous version with supplemental inputs from pseudo-invariant desert sites, providing a more accurate description of the scan-angle dependence. The increased sensitivity of the sensor polarization property manifests itself in the downstream science products and special effort is required to characterize the associated impact. Efforts are also required to examine the potential differences in the SD degradation estimated at the sensor view and SDSM view angle.

The major challenge for the TEB calibration is the PV LWIR crosstalk effects that have gradually increased over time, especially after the recent $(\mathrm{Feb}, 2016)$ safe-hold event ${ }^{15,16}$. This is coupled with that fact that most of the TEB noisy and inoperable detectors are also in these bands. It is clear that a constant monitoring of the crosstalk correction coefficients is necessary along with regular updates to mitigate its impacts on the downstream atmospheric data products. With changes made to the calibration approaches and algorithms, the L1B calibration uncertainties for both RSB and TEB should also be reviewed and updated accordingly.

\section{SUMMARY}

Launched in December 1999, Terra MODIS has successfully operated for more than 16 years. Despite several changes made to its operation configurations, most at the mission beginning, and a few anomalies occurring throughout its mission, which could be related to either spacecraft operation or sensor itself, the Terra MODIS and its on-board calibrators $(\mathrm{OBC})$ continue operate and function normally. Apart from MODIS sensor design improvements over its heritage sensors, jointly dedicated efforts made by the instrument operation, calibration, and science teams have greatly contributed to its mission success. Many science data products of high quality have been generated from Terra MODIS long-term observations. On-orbit calibration coefficients, including sensor gains and response versus scan angle (RVS), have been derived and updated using observations from its OBCs, the moon, and select ground targets with well-characterized properties.

As described and discussed in this paper that the instrument overall performance remains satisfactory in terms of its temperature stability and $\mathrm{OBC}$ functionality. Changes in sensor radiometric, spatial, and spectral characteristics are regularly monitored and calibration updates are made whenever is necessary to support the L1B data production and re-processing. In general, large changes in gains and RVS are observed in the VIS spectral bands. The majority of the noisy and inoperable detectors occurring since launch have been in the LWIR PV bands. These bands have also been impacted significantly by the electronic crosstalk that needs to be characterized and corrected in order to restore the calibration and data quality. Overall spatial and spectral performance of Terra MODIS has been very 
stable. Many lessons learned from its mission-long operation and calibration have benefited other sensors, such as Aqua MODIS and S-NPP VIIRS. As instrument ages, existing and new challenging issues require more devoted effort.

\section{ACKNOWLEDGEMENTS}

Authors in this paper would like to acknowledge contributions made by the MODIS instrument vendor (Raytheon), the Terra flight operation team (FOT), the MODIS instrument operation team (MODIOT), and other calibration and science team members (previous and current) to assist MODIS instrument operation and calibration.

\section{REFERENCES}

[1] W.L. Barnes and V.V. Salomonson, "MODIS: A global image spectroradiometer for the Earth Observing System", Critical Reviews of Optical Science and Technology, CR47, 285-307, 1993

[2] V.V. Salomonson, W.L. Barnes, X. Xiong, S. Kempler, and E. Masuoka, "An overview of the Earth Observing System MODIS Instrument and Associated Data System Performance”, Proc. IGARSS, 1174-1176, 2002

[3] X. Xiong, K. Chiang, J. Esposito, B. Guenther, and W.L. Barnes, "MODIS On-orbit Calibration and Characterization," Metrologia 40 89-92, 2003

[4] Xiong X., J. Sun, W. Barnes, V. Salomonson, J. Esposito, H. Erives, and B. Guenther, "Multi-year On-orbit Calibration and Performance of Terra MODIS Reflective Solar Bands," IEEE Transactions on Geoscience and Remote Sensing, Vol. 45, No. 4, 879-889, 2007

[5] X. Xiong, K. Chiang, A. Wu, W.L. Barnes, B. Guenther, and V. Salomonson, "Multiyear On-orbit Calibration and Performance of Terra MODIS Thermal Emissive Bands," IEEE Transactions on Geoscience and Remote Sensing, 46 (6), 1790-1803, 2008

[6] X. Xiong, N. Che, and W.L. Barnes, "Terra MODIS On-orbit Spatial Characterization and Performance," IEEE Transactions on Geoscience and Remote Sensing, Vol. 43, No. 2, 355-365, 2005

[7] X. Xiong, N. Che, and W.L. Barnes, "Terra MODIS On-orbit Spectral Characterization and Performance", IEEE Transactions on Geoscience and Remote Sensing, Vol. 44, No. 8, 2198-2206, 2006

[8] J. Sun, X. Xiong, W. Barnes, and B. Guenther, "MODIS Reflective Solar Bands On-orbit Lunar Calibration," IEEE Transactions on Geoscience and Remote Sensing, Vol. 45, No. 7, 2383-2393, 2007

[9] X. Xiong, J. Sun, A. Angal, K. Chiang, and W.L. Barnes, “Applications and Results of MODIS Lunar Observations," Proceedings of SPIE - Sensors, Systems, and Next Generation of Satellites XI, Vol. 6744, $67441 \mathrm{H}, 2007$

[10] Sun, J., X. Xiong, A. Angal, H. Chen, A. Wu, and X. Geng, "Time-Dependent Response Versus Scan Angle for MODIS Reflective Solar Bands," IEEE Transactions on Geoscience and Remote Sensing, vol. 52, issue 6, pp. 3159-3174, 2014

[11] X. Xiong, N. Chen, Y. Li, and T Wilson, "Assessments and applications of Terra and Aqua MODIS on-orbit electronic calibration,” Proc. SPIE 9972, Earth Observing Systems XXI, 99720X, doi:10.1117/12.2238603, 2016

[12] Angal, A., X. Xiong, J. Sun, and X. Geng, "On-orbit noise characterization of MODIS reflective solar bands," J. Appl. Remote Sens. 0001; 9 (1):094092, 2015

[13] Madhavan, S., X. Xiong, A. Wu, B. N. Wenny, K. Chiang, N. Chen, Z. Wang, and Y. Li, "Noise Characterization and Performance of MODIS Thermal Emissive Bands," IEEE Transactions on Geoscience and Remote Sensing, vol. 54, issue 6, pp. 3221-3234, 2016

[14] Toller, G., X. Xiong, K. Chiang, J. Kuyper, J. Sun, L. Tan, and W. Barnes, "Status of Earth Observing System Terra and Aqua MODIS Level 1B Algorithm", J. Appl. Remote Sens., vol. 2, no. 023505, 2008 
[15] J. Sun, X. Xiong, S. Madhavan, and B. N. Wenny, “Terra MODIS Band 27 Electronic Crosstalk Effect and Its Removal," IEEE Transactions on Geoscience and Remote Sensing, vol. 52, issue 3, 1551-1561, 2014

[16] T. Wilson, A. Wu, X. Geng, Z. Wang, and X. Xiong, "Analysis of the electronic crosstalk effect in Terra MODIS long-wave infrared photovoltaic bands using lunar images," Proc. SPIE 10004, Image and Signal Processing for Remote Sensing, 2016 